\title{
A Atenção e o Cuidado à Gravidez na Adolescência nos Âmbitos Familiar, Político $e$ na Sociedade: uma revisão da literatura
}

Assistance and Care provided for Teenage Pregnancies in the Family, in Public Policies and in the society: a literature review

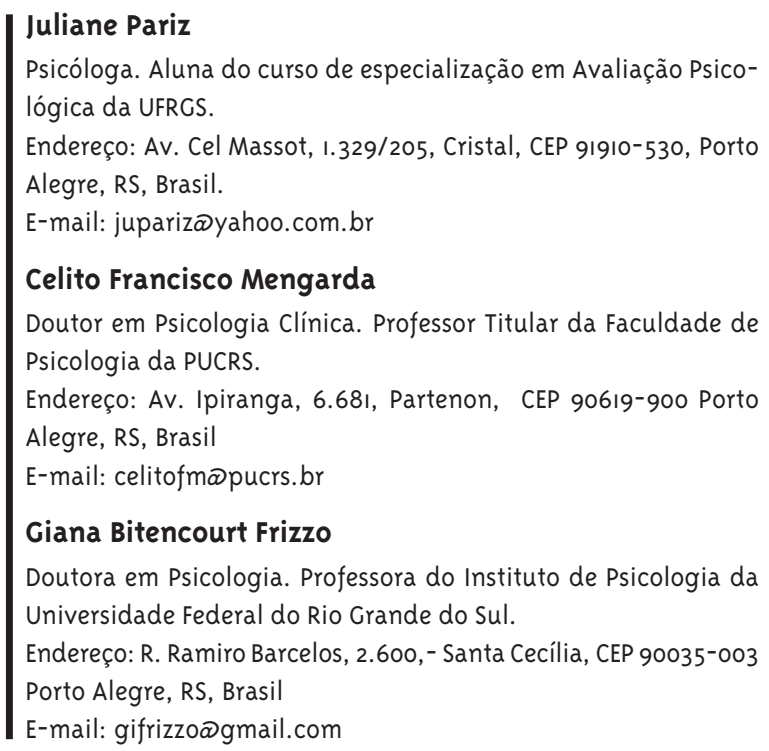

\section{Celito Francisco Mengarda}

Doutor em Psicologia Clínica. Professor Titular da Faculdade de Psicologia da PUCRS.

Endereço: Av. Ipiranga, 6.68I, Partenon, CEP 90619-900 Porto Alegre, RS, Brasil

E-mail: celitofmळpucrs.br

Giana Bitencourt Frizzo

Doutora em Psicologia. Professora do Instituto de Psicologia da Universidade Federal do Rio Grande do Sul.

Endereço: R. Ramiro Barcelos, 2.600,- Santa Cecília, CEP 90035-003 Porto Alegre, RS, Brasil

E-mail: gifrizzoळgmail.com

\section{Resumo}

O presente trabalho pretendeu analisar a questão da gravidez na adolescência, observando o modo como família, política e sociedade têm cuidado dessas jovens. Para tanto, foi realizada uma revisão sistemática da literatura científica nacional com acesso livre nas principais bases de dados entre os anos de 1998 a 2008. Os resultados apontaram dificuldades de comunicação entre cada uma das três esferas estudadas e as adolescentes, fato que vem acarretando um déficit na orientação dos adolescentes que não têm encontrado, na família, na sociedade ou nas políticas públicas a clareza necessária para fazer escolhas mais conscientes e assertivas. Além disso, muitos estudos têm sugerido estratégias de enfrentamento em relação a engravidar durante a adolescência, mas esses achados não têm sido traduzidos em ações ou projetos. Percebe-se que, tanto a adolescência quanto a gravidez nessa etapa da vida, ainda ocupam um lugar confuso na família, política e sociedade e pode-se dizer que, no Brasil, também não está claro o papel de cada uma dessas esferas para atender e cuidar dessa população.

Palavras-chave: Gravidez na adolescência; Família; Sociedade; Política. 


\section{Abstract}

The present article intends to analyze teenage pregnancies observing the ways in which families, policies and society have been taking care of these young women. To achieve this, a systematic review of the national scientific literature was carried out, and the main databases were accessed between the years of 1998 and 2008. The results showed that communication was difficult between each of the three studied spheres and the adolescents, a fact that has produced a deficit in the orientation of these young women, who have not been finding in their family, in society or in public policies, the clarity they need in order to make conscientious and assertive choices. Furthermore, many studies have suggested coping strategies to face difficulties related to teenage pregnancies, although few of the findings have been applied to real actions or projects. It is clear that adolescence, as well as pregnancies during this period, still occupy a confusing position concerning families, policies and society. Particularly in Brazil, the role that each of these spheres play when it comes to taking care of and assisting this population is not clear.

Keywords: Teenage Pregnancy; Family; Society; Policies.

\section{Introdução}

Desde a década de 1970, a maternidade na adolescência vem sendo identificada como um problema de saúde pública. Complicações obstétricas com repercussões para a mãe e o recém-nascido, bem como problemas psicológicos, sociais e econômicos têm fundamentado essa afirmação. As ações voltadas para dar conta dessa temática têm-se apoiado em resoluções baseadas na educação sexual, no acesso a métodos contraceptivos e até mesmo no aborto (Corrêa e Ferriani, 2006; Monteiro e col., 2007; Silva e Tonete, 2006; Lira e Dimenstein, 2004; Altmann, 2007; Yazlle, 2006; Moreira e col., 2008). $\mathrm{Na}$ atualidade, os estudos que identificam as causas mais frequentes para a ocorrência e recorrência da gravidez na adolescência mostram uma contínua relação entre a gestação e o abandono escolar, o apoio da família e o apoio do pai do bebê. Além disso, a ausência de programas de planejamento familiar adequados à demanda dos adolescentes nos serviços públicos de saúde também tem sido discutida como fator importante na etiologia da gestação adolescente (Gonçalves e col., 2001; Godinho e col., 2000; Lima e col., 2004). Contudo, sabe-se que a criação de programas e projetos em saúde pública que sejam específicos para os adolescentes tem sua importância cada vez mais destacada, dadas as consequências sociais e econômicas da gravidez na adolescência e a maior intensidade dos prejuízos de uma atenção precária à gestação nessa fase da vida (Monteiro e col., 2007, Godinho e col., 2000; Lima e col., 2004; Gama e col., 2004).

Assim sendo, percebe-se a importância de um estudo que atente para o que tem sido feito com relação ao crescente número de gestações na adolescência, bem como a forma com que essas ações se apresentam para os adolescentes, ampliando a discussão sobre o modo como se tem dado o cuidado e a atenção à gestação na adolescência nos âmbitos mais citados pela literatura, os quais são: familiar, político e na sociedade.

Para efeito deste trabalho, será considerada atenção à gravidez na adolescência toda estratégia e intervenção que se comprometa com prevenção da gravidez indesejada e também com a saúde das adolescentes durante a gestação. As estratégias e intervenções serão estudadas da seguinte forma: no 
âmbito familiar, serão consideradas todas as intervenções advindas do adolescente ou de sua família, sendo ele a mãe e/ou o pai, que estejam associadas à gestação de adolescentes citadas pela literatura científica publicada entre os anos de 1998 a 2008. No âmbito social, trabalharemos com o conceito de sociedade que, conforme a Teoria Ecológica do Desenvolvimento, proposta por Bronfenbrenner (Papalia e col., 2006), trata-se da "comunidade imediata”, formada por aqueles que estão próximos e íntimos ao sujeito, mas não pertencem ao núcleo familiar, e da Comunidade Institucional, formada pelas instituições que são acessadas pelo sujeito, tal como escolas, grupos, ONGs, igrejas. Segundo o autor, o sujeito encontra-se alocado no centro de um sistema formado por estruturas que atravessam todas as etapas do seu desenvolvimento. Desta forma, estaremos considerando no âmbito que trata da sociedade, a influência da "comunidade imediata" e da "comunidade institucional", ou seja, todo o apoio e o cuidado que ocorrer fora da família e à parte de uma política pública de saúde como, por exemplo, o apoio promovido pelas escolas e por amigos.

No âmbito político, nosso foco serão as estratégias elaboradas e estruturadas pelas políticas públicas e privadas e que são operacionalizadas por meio dos programas e serviços de saúde, para atender à temática específica da gravidez na adolescência citadas pela literatura científica publicada.

\section{Método}

Revisões sistemáticas são investigações científicas que seguem um método para buscar e selecionar nas pesquisas, usando critérios de inclusão e exclusão. É uma forma de síntese das informações disponíveis em dado momento sobre um tema específico (Otoni e Barros, 2011). Mesmo assim, as revisões sistemáticas têm grande importância como ferramenta de investigação científica para a tomada de decisão, além de custos bem menores do que os exigidos para a realização de estudos de larga escala (Berwanger e col., 2007).

A pesquisa objetivou revisar a literatura publicada on-line dos últimos dez anos - isto é, de 1998 a 2008 - em artigos científicos com acesso livre nas seguintes bases de dados: Pub Med (localizado um total de 180 artigos), Scielo (localizados em torno de 230 artigos), Portal Capes (localizados em torno de 200 artigos). Foram utilizados os descritores - aqui, em ordem decrescente de localização de artigos: Teenager and pregnancy (em torno de 160 artigos); gravidez and sociedade (em torno de 145 artigos); Adolescência and gravidez and família (em torno de 115 artigos); adolescente and política (em torno de 100 artigos); adolescence and family (em torno de 115 artigos).

Dos artigos encontrados, foram incluídos os estudos sobre o cuidado e atenção ao planejamento familiar na adolescência feitos no Brasil e que evidenciaram as intervenções que têm sido feitas com relação a essa temática nos âmbitos: Familiar (orientação e preparo dos pais e mães e companheiros das adolescentes), Político (Programas governamentais operacionalizados sob a forma de programas de saúde e de inserção nas escolas), Na sociedade (Projetos não governamentais e intervenções de pessoas com algum vínculo com a temática) restando assim, de todos os artigos localizados, em torno de 31 trabalhos para a realização desta contribuição. Foram excluídos os estudos que traziam percepções e pontos de vista a respeito da gravidez na adolescência e/ou do uso de métodos anticoncepcionais; que relacionaram gravidez e problemas orgânicos; que não consideraram exclusivamente a adolescência e o que se tem feito quando uma adolescente engravida. Estudos específicos sobre o aborto ou sobre doenças durante a gestação adolescente também foram excluídos. Estudos sobre a utilização dos métodos anticoncepcionais foram considerados apenas quando os dados constaram de pesquisas demográficas e/ou epidemiológicas.

Dentre os artigos revisados e citados neste trabalho, não foi possível destacar algum autor específico. Pensamos que isso tenha ocorrido devido ao fato de, na literatura atual, não haver um costume em integrar os âmbitos dentro de uma temática, tal como é a proposta deste artigo. Assim, agregamos aqui uma grande diversidade de autores dentro de cada âmbito estudado.

A análise do material foi feita através dos seguintes eixos norteadores: contextualização de adolescência, aspectos familiares, aspectos políticos e aspectos da sociedade, resultados e discussão. 


\section{Contextualização da Adolescência}

A definição de adolescente realizada pela Organização Mundial de Saúde (OMS) inclui aspectos biológicos, sociais e psicológicos e delimita a faixa etária da adolescência com uma definição cronológica, propondo que esse período se estenda dos 10 aos 19 anos de idade. Entretanto, o Estatuto da Criança e do Adolescente (ECA) (1990) propõe que a adolescência seja o período compreendido entre 12 e 18 anos de idade de uma pessoa.

Conforme Berlofi e colaboradores (2006), na adolescência desenvolvem-se processos psicológicos e padrões de identificação que evoluem da fase infantil para a adulta, entre eles a transição de um estado de dependência para outro de relativa autonomia. Sabe-se também que esse período é caracterizado por mudanças físicas e hormonais que, por si só, exigem do ser humano em transformação um trabalho psíquico que dê conta da elaboração do luto do seu corpo e seus hábitos da infância para assumir um novo corpo, que se impõe com novas exigências de cuidado e comportamento transportando o adolescente para o lugar que ocupará na sociedade.

Ao estudar as intercorrências da adolescência, especialmente a gestação nessa fase da vida, a literatura frequentemente utiliza o termo vulnerabilidade para designar o estado em que se encontram os grupos de indivíduos fragilizados, social e politicamente, quanto à promoção, proteção ou garantia de seus direitos de cidadania. Neste sentido, conforme Ferrari e colaboradores (2008), o adolescente é considerado vulnerável por ser parte de um grupo social que se encontra em fase de importantes transformações biológicas e mentais, articuladas a um redimensionamento de identidades e de papéis sociais.

Em meio a estas transformações físicas e psíquicas, quando ocorre a gestação na vida de adolescentes, esta gestação trará consigo profundas e abrangentes mudanças nos aspectos físicos e psicológicos, com repercussões individuais, familiares e sociais. Em função disso, desde as décadas de 1980 e 1990, o adolescente foi reconhecido pela sociedade da América Latina e Caribe como foco de estudo no campo da Saúde Pública (Lira e Dimenstein, 2004, Ferrari e col., 2008; Damian, 2003).
Contudo, é importante considerar que, ao contrário da visão hegemônica da sociedade e da saúde pública em geral, que considera gravidez na adolescência como indesejada, a maternidade na adolescência tem se mostrado como uma experiência de vida que também pode trazer significados positivos (Santos e Schor, 2003); ainda que devemos reconhecer como legítimas muitas das preocupações da família e da sociedade, é preciso entender que a concepção negativa e reducionista sobre a gravidez na adolescência pode construir restrições e implicações conceituais no desenvolvimento de pesquisas e na atuação dos profissionais junto aos adolescentes. Por isso, um melhor entendimento das circunstâncias possibilitará que os profissionais de saúde planejem e executem ações de saúde mais adequadas e eficientes para essa população (Santos e Schor, 2003).

Apoiando as ideias de Santos e Schor (2003), outro autor sustenta que a gravidez pode ser bem tolerada pelas adolescentes, desde que elas recebam assistência pré-natal adequada, ou seja, precocemente e de forma regular durante todo o período gestacional, o que nem sempre acontece (Yazlle, 2006). Tal fato é atribuído por este autor a vários fatores que vão desde a dificuldade de reconhecimento e aceitação da gestação pela jovem até a dificuldade para o agendamento da consulta inicial do pré-natal (Yazlle, 2006).

Ao se descobrir grávida, a adolescente enfrenta inúmeras dificuldades dependendo, sobretudo, de sua classe social (Godinho e col., 200o; Heilborn e col., 2002); e são estas dificuldades, aliadas ao entendimento da realidade de cada comunidade, bem como o conhecimento a respeito das mudanças e particularidades implicadas na adolescência que poderá dar aos familiares, à sociedade e aos programas e projetos políticos os subsídios necessários para a adoção de medidas mais assertivas na atenção à adolescência e à gestação na adolescência.

\section{A Atenção e o Cuidado no Âmbito Familiar}

A respeito da atenção e do cuidado da família com sua adolescente, a literatura aponta modificações com a descoberta da gravidez. No estudo de Monteiro 
e colaboradores (2007), as adolescentes relataram que "viviam uma relação boa, mas ao revelarem que estavam grávidas foram vítimas de atos violentos, como violência física e psicológica, sendo discriminadas e culpabilizadas por parte dos pais" com os quais o diálogo sobre o assunto ainda permanece distante (Monteiro e col., 2007, p. 373). As jovens também relataram que frequentemente sofreram críticas de familiares, seja pelas pressões sociais envolvidas, seja por problemas financeiros.

Ademais, muitas vezes não podem contar com o apoio de amigos ou vizinhos, sentem-se envergonhadas, culpadas e têm dúvidas quanto ao seu futuro e ao de seu filho. Quando as adolescentes percebem que estão grávidas, elas recorrem, primeiramente, ao parceiro, depois a sua mãe e em seguida aos amigos, sendo que, habitualmente, a comunicação pode ser mais bem estabelecida com a mãe (Godinho e col., 2000).

Sabe-se, porém, que as reações da família diante da adolescente grávida tendem a ser paradoxais dada a sobreposição dos sentimentos de revolta, abandono e aceitação do inevitável (Lima e col., 2004), podendo esses sentimentos ser transformados ou não em aceitação e apoio, dependendo da forma como a família compreende a gravidez de sua adolescente (Lima e col., 2004). Existe uma vasta extensão de estudos que reconhecem que o apoio familiar é muito importante para vivenciar uma gestação, especialmente na adolescência. Ainda assim, muitas famílias podem sentir um "choque pela notícia” que vem acompanhado por um sentimento que pode ser de impotência quanto à prevenção da gravidez, seguido de um conformismo; como também de alegria e melhora no relacionamento familiar com a chegada do bebê (Silva e Tonete, 2006). De modo geral, a gestação da adolescente vem para a família carregada com um grande impacto emocional. É possível evidenciar, em alguns casos, uma frustração devido à interrupção/mudança no projeto de vida familiar em relação à adolescente, especialmente quando não houver um relacionamento estável com o pai da criança (Silva e Tonete, 2006).

Pode-se dizer que a família encontra dificuldades em cuidar e apoiar a gestação de sua adolescente. Dentre essas dificuldades, a que mais se destaca é a comunicação, ou seja, a ausência ou a inabilida- de para conversas sobre sexualidade com filhas e filhos (Monteiro e col, 2007; Dias e Gomes, 1999). Dentre os fatores apontados como complicadores para a expressão correta das orientações dentro das famílias, destacam-se a confusão de valores e informações ambíguas (Monteiro e col., 2007; Dias e Gomes, 1999). A origem desta dificuldade por parte dos pais pode estar na troca do modelo hierárquico, no qual os papéis familiares eram rigidamente estabelecidos e o poder centralizado na figura do pai, por um modelo igualitário, no qual se destacam os ideais de liberdade e respeito à individualidade, os quais ainda não estão muito especificados atualmente (Dias e Gomes, 1999). Neste novo modelo, o certo e o errado se confundem para os pais enquanto seus filhos passam a ser orientados pela experimentação e descoberta, ficando sujeitos, dentre outras coisas, à gravidez ainda durante a adolescência (Dias e Gomes, 1999). Neste sentido, pode ser preciso voltar o olhar para a necessidade de um trabalho de reorientação de valores para que, mais seguros e melhor orientados, os pais possam dar conta de algumas ansiedades e inabilidades dos filhos adolescentes e estes estejam mais bem preparados para fazer escolhas tão importantes como ter um filho, por exemplo.

Dentro da dimensão familiar, a ausência do companheiro tem sido apontada como um complicador social e obstétrico decorrente da gravidez, pois a recusa da paternidade pode ser fonte de estresse para a adolescente, tornando-a vulnerável a complicações perinatais, no parto e na saúde da criança (Gonçalves e col., 2001; Gama e col., 2004), ao passo que a presença do companheiro pode influenciar favoravelmente na evolução da gravidez e diminuir os riscos e efeitos físicos e psicológicos desfavoráveis à saúde da criança e da mãe (Gama e col., 2004; Costa e col., 2005). Entretanto se faz importante considerar que o pai adolescente pode sofrer uma reação negativa do meio que o rodeia sendo constantemente censurado e, na maioria das vezes, condenado. Além disso, há necessidade imediata de o pai contribuir financeiramente quase sempre sem estar preparado para isso. Neste sentido, é importante ressaltar que a dificuldade ou impossibilidade de o pai contribuir financeiramente durante a gravidez ou criação do filho tem sido entendida e aceita pelas adolescentes, 
ao passo que a ausência, o não envolvimento e a falta de apoio emocional, não.

O modo de apoio recebido do companheiro tem variado desde aqueles que ofereceram todo tipo de ajuda material até aqueles que deram conforto e carinho (Gonçalves e col., 2001). Não obstante todo o avanço da medicina, são estas variáveis psíquicas e sociais de apoio e aceitação familiar que se têm mostrado mais decisivas para os melhores prognósticos pré e perinatais (Gama e col., 2004; Costa e col., 2005).

\section{A Atenção e o Cuidado no Âmbito das Políticas Públicas}

Partindo da compreensão de que as políticas públicas e privadas operacionalizam-se por meio dos programas e serviços de saúde, torna-se necessário atentar para o contexto e as premissas que envolvem a estruturação desses serviços. A Constituição de 1988 gerou profundas modificações na organização das políticas públicas do País. Desde então, os sistemas de saúde vêm passando por transformações ao longo do tempo no sentido de atender às necessidades de seus usuários, bem como aos interesses políticos, sociais e econômicos. Naturalmente, a capacidade de um país para gerar riquezas vai depender da formação profissional dos membros de sua sociedade - os adolescentes estão no meio desse processo de capacitação (Baraldi e col., 2005) e é desejável que eles completem a sua formação educacional e profissional, integrem o mercado de trabalho e, a partir desse momento, colaborem gerando a prole que vai garantir a continuidade de seu grupo social. A partir desse raciocínio, percebe-se que a prevalência de mães adolescentes de baixa renda, com limitações profissionais, faz crescer também a demanda pelos serviços de saúde das crianças deste grupo social (Baraldi e col., 2005).

A partir desses dados, algumas tentativas de atender aos adolescentes, especialmente os de baixa renda, tem surgido pelo Brasil. No ano de 1989, foi implantado o Programa de Saúde do Adolescente (PROSAD) para meninos e meninas de 10 a 19 anos de idade. 0 objetivo do programa era a atender a sexualidade e a saúde reprodutiva, além de estabelecer os direitos dos adolescentes por intermédio do Estatuto da Criança e do Adolescente (ECA). Em sua essência, tratou-se de uma proposta de atenção integral à saúde do adolescente enraizada no paradigma biológico e centrada no conceito de risco, alegando incapacidade fisiológica para gestar e incapacidade psíquica para criar, abordando a gravidez na adolescência como um problema de saúde pública que impediria o cumprimento da função social dos jovens (Ferrari e col., 2008; Santos e Schor, 2003; Heilborn e col., 2002; Vieira e col., 2006). Mesmo assim, o PROSAD não se mostrou suficiente, os dados referentes à gravidez indesejada e não planejada na adolescência vêm demonstrando índices alarmantes ano após ano e as políticas públicas para esse grupo etário continuam fragmentadas e desarticuladas, mesmo existindo inúmeras iniciativas. 0 que nos permite inferir, a respeito dessas iniciativas, que elas não têm representado significativamente um trabalho intersetorial para a integralidade da atenção de que esse grupo necessita (Ferrari e col., 2008).

No período de 1994-1996, o Serviço multiprofissional de Saúde Emaús prestava atendimento clínico e planejamento familiar para adolescentes, pré-natal para gestantes adolescentes e acompanhamento clínico dos filhos de adolescentes (Costa e Formigli, 2001). Esses autores, ao avaliarem tal projeto, indicam que é preciso levar em conta aspectos específicos da adolescência para viabilizar o conhecimento da real situação de saúde dos adolescentes. É interessante perceber que o projeto "Afetividade, Sexualidade e Regulação da Gravidez na Adolescência”, desenvolvido atualmente e organizado pelo UNI/Natal e pela Secretaria Municipal de Saúde da Cidade de Natal/RN, com o objetivo de reduzir a gravidez entre adolescentes e garantir a assistência ao pré-natal utilizando o atendimento multiprofissional e a criação de novos protocolos de intervenção (Lira e Dimenstein, 2004), também foi avaliado e, como o projeto Emaús, sua avaliação mostrou um grande complicador da qualidade da atenção à adolescência, possivelmente devido a um confronto entre a percepção e a realidade dos adolescentes e a dos profissionais quanto ao que seja adolescência, conforme criticado por Lira e Dimenstein (2004). A maioria dos adolescentes sente-se capaz de responsabilidade, conhecimento e liberdade de escolha, sonhos, descoberta do ser cidadão, enquan- 
to o profissional, na visão do adolescente, expressa exatamente o contrário.

Acredita-se que isto pode acarretar consequências, principalmente no que se refere à autoestima e à adesão ao programa, porque todos necessitam do reconhecimento de seu valor (Lira e Dimenstein, 2004).

Essas avaliações também revelaram resultados satisfatórios com relação à qualidade técnico-científica do atendimento oferecido a adolescentes em serviços de saúde: avaliação pediátrica, pré-natal, intervalo entre consultas, vacinação, registros gerais de saúde. Os resultados menos satisfatórios permaneceram nas condutas clínicas com adolescentes, sendo estas total ou parcialmente inadequadas (Costa e Formigli, 2001). Para tornar satisfatório o atendimento clínico aos adolescentes é importante levar em conta aspectos específicos da adolescência que podem ocorrer com a maioria deles: desconsideração das situações de risco e a dificuldade de lidar com os conflitos decorrentes da gestação (razão pela qual necessitam de acompanhamento periódico e atenção específica). Neste sentido, a capacitação da equipe torna-se indispensável para viabilizar o conhecimento da real situação de saúde dos adolescentes. Uma importante sugestão que tem surgido na literatura é a avaliação periódica que é factível de realização na rotina dos serviços de saúde para a constante reorientação das práticas a fim de atingir melhores resultados (Damian, 2003; Costa e Formigli, 2001).

Outras intervenções que têm surgido são postos de saúde em que as agentes comunitárias fazem visitas domiciliares para orientar adolescentes e familiares sobre contracepção e doenças sexualmente transmissíveis. Esse trabalho tem demonstrado resultados positivos, mas ainda se faz necessário ultrapassar determinados obstáculos tais como o já citado preparo dos profissionais de saúde para tratar da temática e o planejamento adequado das ações, pois na ausência de programas específicos para adolescentes na unidade de saúde, os estudos têm demonstrado que os profissionais procuram atender essa população da melhor maneira possível com os programas já existentes, mas isso tem gerado sobrecarga de trabalho e necessidade de revisão das intervenções que encontram a predisposição das equipes para implantar programas específicos de atenção à saúde do adolescente (Lima e col., 2004; Ferrari e col., 2008).

Nos últimos anos, também foram criados programas de assistência pré-natal específicos para esse grupo, os quais buscam, através de uma equipe multidisciplinar, implementar uma assistência pré-natal integral e incrementar a autoestima. Entretanto, uma parte das adolescentes não tem tido acesso a esses programas, ainda escassos no País (Godinho e col., 200o). Mas esse acesso deve ser entendido por dois lados: de um, as facilidades ou dificuldades que os usuários vivenciam para se aproximarem do serviço; de outro, as características do serviço no que tange à compatibilidade dos horários de atendimento, disponibilidade, preparo e acolhimento por parte dos profissionais (Carvacho e col., 2008). Esse conceito ampliado de acesso precisa ser mais abordado para facilitar o entendimento das dificuldades que os adolescentes enfrentam para utilização do serviço de saúde (Carvacho e col., 2008), pois as dificuldades de acesso agravam as dificuldades já existentes de educação, informação, prevenção e cuidado. Dentre as barreiras de acesso ao serviço de saúde, estudos mostram que as psicossociais são as mais significativas, sendo elas: deslocamento e acolhimento nos serviços, barreiras de gênero - destacando-se o sentimento de vergonha no atendimento por ginecologista do sexo masculino - e ações que desconsideram características sóciodemográficas das adolescentes, bem como o vínculo com seus parceiros (Carvacho e col., 2008).

Outros dados importantes a respeito dos serviços já existentes mostraram que as orientações fornecidas são interpretadas como uma forma de apoio durante a gravidez no que se refere à prevenção (Godinho e col., 2000; Damian, 2003). Entretanto, a maioria dos programas de saúde da família não têm conseguido, até o momento, realizar um trabalho de educação sexual entre os adolescentes (Borges e col., 2006). Mesmo assim, estudos indicam que boa parte dos adolescentes recebe informações sobre contracepção, sendo a pílula e o preservativo os mais conhecidos e utilizados. Porém, registram-se elevada inadequação na utilização dos métodos contraceptivos, evidenciando a falta de serviços devidamente aptos para transmitir orientações e 
atendimento adequado aos adolescentes (Vieira e col., 2006).

A análise da compreensão dos profissionais quanto às práticas de atenção aos adolescentes indica que as ações programáticas, preventivas e de intervenção são realizadas mais pelos enfermeiros do que pelos médicos, que esgotam seus atendimentos na consulta. Ambos afirmam orientar com relação à prevenção da gravidez, DST/AIDS e uso do preservativo, entretanto todos concordam que é preciso "capacitar todos os profissionais, reestruturar os recursos materiais e humanos, inserir outros profissionais e integrar as ações com serviços, além do da saúde" (Ferrari e col., 2008, p. 388). Contribuindo para esta percepção, a literatura tem apontado caminhos para nortear a construção de estratégias de prevenção e atenção à gravidez, deixando clara a necessidade não só de ampliar o acesso a serviços especializados, como também a importância de se utilizar um processo educativo de forma contextualizada com os conhecimentos e realidades pertinentes a cada local a fim de promover o desenvolvimento dos recursos próprios do contexto familiar e social em que se encontram (Silva e Tonete, 2006; Lima e col., 2004; Berlofi e col., 2006; Ferrari e col., 2008; Damian, 2003; Vieira e col., 2006; Siqueira e col., 2002).

Estas novas estratégias também devem conceber a paternidade na adolescência como parte integrante do processo gestacional dada a importância social, familiar e econômica do pai na gestação. Esta concepção favoreceria o surgimento e a organização de serviços de saúde, tendo em vista que, em linhas gerais, eles não vêm sendo estruturados de forma a inserir o pai adolescente na proposta de assistência pré-natal (Corrêa e Ferriani, 2006; Costa e col., 2005; Orlandi e Toneli, 2008). Outro aspecto que aparece com relevância é o fato de algumas gestações na adolescência serem, na verdade, desejadas. 0 querer não tem sido problematizado pelos programas investigados, ao contrário, parte-se do suposto de que a gravidez é indesejada, inconsequente, fruto da irresponsabilidade, da imaturidade e da impulsividade desses jovens. Por que a gravidez na adolescência é assim significada na contemporaneidade? É este o questionamento que se faz cada vez mais necessário para a elaboração de políticas públicas mais novas e eficazes nesta área (Siqueira e col., 2002).

Com isto, postula-se que a estruturação de políticas públicas que se proponham a dar conta da gravidez na adolescência deve atentar para a necessidade de oferecer atendimento e acompanhamento integral antes mesmo do início da vida sexual, mobilizando a sociedade a desenvolver e praticar uma postura crítica, consciente e responsável no exercício da sua sexualidade e de seus adolescentes, além de capacitação dos serviços e profissionais que, a partir de equipes treinadas especialmente para as particularidades desse grupo, garantam reflexos imediatos na saúde reprodutiva de toda a população (Berlofi e col., 2006; Baraldi e col., 2005; Vieira e col., 2006; Oliveira, 1998; Simões e col., 2003). Da mesma forma, deve ser incorporado pelos gestores de políticas públicas o dado que mostra que a fecundidade tende a diminuir com o aumento da escolaridade e do nível de rendimento (Berlofi e col., 20o6), ou seja, o incremento da educação apresenta reflexos imediatos na saúde sexual e reprodutiva da população (Oliveira, 1998). Ainda com relação ao planejamento de políticas públicas, é importante considerar que os estímulos ambientais têm propiciado a iniciação sexual de forma cada vez mais precoce e que a gravidez na adolescência aparece como principal consequência desta iniciação (Berlofi e col., 2006). Estudos sugerem uma retomada da questão moral que está hoje obscurecida por inquietações sobre o impacto do sexo na qualidade de vida do adolescente e merece urgente e despreconceituosa atenção (Lira e Dimenstein, 2004).

Mais recentemente, a Estratégia da Saúde da Família - ESF tem-se mostrado como o ponto de partida mais propício para redirecionar as ações programáticas até então instituídas para o grupo de adolescentes nas diferentes áreas de abrangência dos serviços de atenção básica de saúde.

Estudos têm sugerido que o profissional de saúde que atua na atenção básica no seu campo de abrangência pode estar mais capacitado para apreensão do quadro de vulnerabilidades locais, inclusive compreender a dimensão concreta da vida do adolescente no processo saúde-doença, subsidiando a prática educativa de alcance coletivo em educação e em saúde e dando conta das várias formas de relação dos adolescentes nas esferas da vida nas cidades, da 
cultura, do trabalho, da instituição educacional, das relações familiares, da sexualidade, do lazer e da Constituição Brasileira (Ferrari e col., 2008).

Como podemos perceber, estruturar políticas públicas para atender à gravidez na adolescência é, em suma, atuar intersetorialmente, ou seja, considerando as implicações na saúde e na educação, com tudo o que está atrelado a cada uma dessas áreas.

O surgimento de pílulas anticoncepcionais, programas de planejamento familiar e um controle definitivo sobre o número de filhos, com a realização de esterilizações de homens e mulheres jovens, aparecem como as estratégias mais implementadas, mas apesar de todo o cuidado e discurso médico, é possível afirmar que o principal desafio imposto para as políticas públicas nesta área está em atingir um grande contingente de adolescentes a cada estratégia planejada (Souza, 2006).

Diante disso, a escola torna-se um local privilegiado para expansão da educação sexual. "Daí a recorrente evocação do papel que esta tem a exercer sobre essa problemática social." (Altmann, 2007). Justificada a necessidade de a escola participar da orientação sexual devido aos aumentos dos casos de gravidez e de DST/AIDS entre jovens, foi criada, nos Parâmetros Curriculares Nacionais (PCN), a inserção transversal do assunto como medida de prevenção e de promoção de saúde para os estudantes. Na prática, são os livros didáticos de ciências os que mais se aproximam da temática e, com dificuldade de implementação, a proposta inicial aparece muito mais como um ideal, do que como o que é de fato realizado no dia a dia da escola (Altmann, 2007). Estudos apontam que a referida dificuldade ou até mesmo a ausência de educação sexual nas escolas pode ser um dos fatores associados à ocorrência de gravidez indesejada na adolescência (Godinho e col., 200o). Entretanto, torna-se difícil avaliar o papel da escola, tendo em vista que a grande maioria das adolescentes abandona os estudos após a gravidez apesar de referirem que a escolarização constitui parte de um projeto de realização também pessoal, ocupando, junto com a maternidade, um lugar central nas suas vidas (Godinho e col., 200o; Pantoja, 2003).

\section{A Atenção e o Cuidado na Sociedade}

A sexualidade adolescente tem sido focada como um problema para a sociedade (Altmann, 2007). É o que dizem alguns dos estudos que falam a respeito da gravidez na adolescência que, na realidade cultural brasileira, não é tida como uma experiência a ser vivenciada nesta fase da vida. Por isso, diferentes campos como a medicina, a demografia e a educação articulam-se com o intuito de gerir a sexualidade adolescente a fim de, entre outras coisas, evitar a gravidez (Altmann, 2007).

Torna-se relevante o esclarecimento de que, ao tratarmos de sociedade, estamos aqui contextualizando todas as ações que objetivam atender e cuidar da gestação adolescente, mas que são oriundas das redes de sociabilidades que, nesta pesquisa, entendemos que se enquadram nos conceitos de "comunidade imediata" e "Comunidade Institucional” propostos por Bronfenbrenner (Papalia e col., 2006). Ou seja, genuinamente se estruturaram e se operacionalizaram a partir de pessoas que foram ou estão conectadas e/ou sensibilizadas de alguma forma com esta temática e que não fazem parte do núcleo familiar: amigos, ONGs etc. Desta forma, queremos diferenciar as ações da sociedade das ações sociais, pois esta última é mais abrangente e está contida dentro do âmbito político.

Para verificar com profundidade a atenção e o cuidado à gravidez na adolescência na sociedade, será necessário passar por alguns caminhos. Estes caminhos estão impregnados com ideias que há anos embasam toda a mobilização, planejamento e até a culpabilização em torno da temática. Primeiramente, a literatura aponta para o fato de "a sexualidade ser um importante foco de investimento político e instrumento de tecnologia de governo" (Altmann, 2007). Durante um período da história, “o Estado dependia do rápido crescimento da mão de obra para concretizar sua expansão e impulsionar as grandes transformações da época" (Souza, 20o6). Entretanto, a partir do movimento de contracultura na década de 1960, os jovens passaram a reivindicar o direito ao livre exercício da sexualidade, fazendo com que a gravidez passasse a ocorrer fora dos laços matrimoniais, resultando num preocupante crescimento 
populacional que se tornou um problema quando somado ao processo maciço de industrialização que começava a "dispensar" o trabalho humano, criando excedente de mão de obra e muitas preocupações para o Estado (Souza, 2006). Com o passar dos anos, o controle da sexualidade tem-se mostrado cada vez mais fundamental para o controle social que, para ser eficaz, precisará prever e prover, entre outras coisas, os investimentos em políticas sociais que garantirão a sobrevivência dos adolescentes e seus filhos criados predominantemente em comunidades pobres. Além disso, estão igualmente implicados neste controle o discurso médico e suas razões de saúde pública, a relação custo-benefício para o Estado, bem como alguns estudiosos salientando que a gravidez entre jovens tem sido mais recorrente em “comunidades pobres" e "populações mais carentes", prevendo o desdobramento de uma cadeia de problemas sociais, na medida em que aumenta esta camada da população, dificultando ainda mais a atenção e a erradicação da pobreza (Altmann, 2007).

Atualmente é possível perceber que a adolescente no seu novo papel de mãe precisa ocupar um novo lugar tanto na comunidade em que vive quanto na sociedade. Esta socialização é realizada, simultaneamente, pela família, pela escola, pela igreja, pela mídia e pelo grupo de iguais (Lima e col., 2004; Pantoja, 2003). Perguntados a respeito de quem mais os tem ajudado, os pais adolescentes apontam os amigos, quando o assunto for o sexo. Apesar disso, os pares foram perdendo prioridade de acordo com a "complexidade" do assunto a ser abordado, sendo mais citados os professores e profissionais de saúde quando as dúvidas diziam respeito à prevenção de DST/AIDS (Borges e col., 2006). 0 mesmo estudo ainda mostra que os pais foram referidos por aproximadamente $20 \%$ dos adolescentes como fonte de esclarecimento de dúvidas, independentemente do assunto abordado (Borges e col., 2006). 0 importante nestes dados é percebermos que todos esses sujeitos necessitam ser "agregados como partícipes das ações de promoção da saúde sexual e reprodutiva de adolescentes" (Borges e col., 2006), isto porque o cuidado à gestação na adolescência deve se constituir em uma ação coletiva ao invés de focalizar apenas a responsabilidade individual. A despeito desta ação coletiva, alguns estudos têm sugerido que as ações de planejamento familiar devem ser divulgadas por meio de diálogos em escolas, centros comunitários, unidades de saúde e reuniões com diferentes grupos etários (Vieira e col., 2006), sempre utilizando materiais de divulgação adequados para cada grupo específico, sugerindo também a importância da utilização dos meios de comunicação em massa (Vieira e col., 2006).

É importante perceber o que ocorreu em algumas estratégias que atingiram resultados esperados com relação à diminuição da gestação na adolescência, como é o caso de campanhas baseadas na sensibilização e educação do adolescente que alcançaram, depois de 15 anos de realização, a redução dos índices de gestação na adolescência na Holanda (de 34/1.ooo adolescentes para 7/1.ooo) e nos Estados Unidos (de 120/1.0oo para 54/1.0oo adolescentes) (Vieira e col., 2006). Esses programas sugerem que as tentativas de prevenção devem levar em consideração o conhecimento dos chamados fatores predisponentes ou situações precursoras da gravidez na adolescência, tais como: baixa autoestima, dificuldade escolar, abuso de álcool e drogas, comunicação familiar escassa, conflitos familiares, pai ausente e/ ou rejeitador, violência física, psicológica e sexual, rejeição familiar pela atividade sexual e gravidez fora do casamento. Ainda têm sido referidos: separação dos pais, amigas grávidas na adolescência, problemas de saúde e mães que engravidaram na adolescência. É importante perceber que alguns estudos sugerem que entre as adolescentes que não engravidam, os pais têm melhor nível de educação, maior religiosidade e ambos trabalham fora de casa (Yazlle, 2006).

$\mathrm{Na}$ atualidade o exercício da sexualidade tem seu início cada vez mais cedo, impulsionado por uma imposição social que leva crianças a adolescerem precocemente e, de forma semelhante, leva os adolescentes a rapidamente ingressarem na vida adulta mesmo não estando preparados psicologicamente. Dessa forma, a sexualidade e a gravidez na adolescência podem ser pensadas pelos diferentes valores, atitudes e padrões de comportamentos existentes na sociedade moderna (Moreira e col., 2008). Dentro desse contexto de impulso social culminando em impulso sexual, espera-se que existam iniciativas, serviços e campanhas que orientem os jovens so- 
bre seus problemas, conflitos ou questionamentos cotidianos durante essa fase de descobertas e modificações em todos os níveis, mas infelizmente, esses recursos informativos são raros nos serviços públicos e até mesmo nos privados como as escolas, os projetos sociais focalizados nesse período da vida, entre outros. O direcionamento de diversos fatores como o desconhecimento do corpo, a omissão da família/escola sobre assuntos pertinentes à adolescência, o pouco envolvimento dos serviços públicos, o bombardeamento ativo ao qual estão expostos pela mídia, com programas, novelas e até propagandas apelando ao sexo, fazem com que os jovens iniciem precocemente suas atividades sexuais, não cônscios das implicações de sua vida sexualmente ativa (Moreira e col., 2008).

\section{Discussão}

Ao discutir a respeito dos dados encontrados, veremos que voltar o olhar para a atenção e o cuidado à gravidez na adolescência não é uma tarefa fácil. Assim, para alcançar uma visão mais ampla da temática, discutiremos os três âmbitos estudados, agrupando-os. Ao optar por esta forma de discussão, é possível de início perceber o predomínio de duas visões opostas entre tantas: a dos adolescentes indicando que nem sempre a gravidez é um problema ou indesejada e a de muitos pesquisadores, políticos e sanitaristas apontando o fato como um problema de saúde pública e movimentando-se para o estabelecimento de programas e projetos que deem conta da prevenção da gravidez adolescente. Além disso, as famílias de forma geral também identificam a gravidez na adolescência como um problema. Inicialmente é preciso propor uma reflexão: as ações estabelecidas atualmente têm dado conta da adolescente grávida - como sujeito em transformação e agente transformador da sociedade em que vive - ou somente estão interessadas em atender a gravidez da adolescente?

Analisando o panorama dos últimos dez anos, foi possível perceber pontos semelhantes nos diversos âmbitos estudados: No âmbito familiar, grande parte das famílias não gosta de conversar sobre o assunto, não esperam que isto aconteça em sua casa e sofrem um intenso impacto emocional a partir da notícia.
Tal impacto frustra a família e causa uma sobreposição de sentimentos contraditórios relacionados à adolescente, piorando o que já se mostra deficitário em grande parte das famílias, a comunicação. Sabese que a orientação vinda da família poderia ser decisiva para a ocorrência de gravidez em adolescentes, entretanto, estudos mostram que uma das maiores dificuldades nesta área está em uma mudança de modelo familiar, pois ao longo dos últimos anos, partiu-se de um modelo hierárquico em que os pais sabiam o que fazer, para um modelo igualitário que tem confundido o papel dos pais que, sem saber o que fazer, não conseguem manejar suas ansiedades, tampouco as inabilidades dos filhos. Estes jovens, sem mínima orientação ou preparo, passam a se guiar pela experimentação e pela descoberta, podendo assim deparar-se, entre outras coisas, com uma gravidez - que pode, ou não, ser indesejada. Não se pode simplificar uma questão tão complexa, mas, a partir dos dados expostos neste estudo, é possível pensar que um trabalho que discuta e reoriente valores para as famílias surge como uma necessidade urgente nos dias atuais, espacialmente no âmbito da saúde pública, já que não foram encontrados, ao longo desta pesquisa, programas e/ou projetos que auxiliem as famílias de adolescentes de forma mais consistente.

Analisando a literatura para o âmbito político, fica evidente que, nos últimos anos, as políticas para gestantes adolescentes tem-se concentrado no atendimento pré-natal, - que realmente é importantíssimo - mas somente este atendimento não dá conta da real necessidade das adolescentes, tampouco da legislação que, conforme a constituição de 1988, deixa claro que a atenção à saúde do adolescente precisa dar-se de forma integral, contemplando todos os aspectos que envolvem e cercam a vida e afetam a saúde física e mental do sujeito. Mesmo assim, foi possível perceber que há algumas iniciativas que têm tido bons resultados, mas ainda será necessário muito trabalho em conjunto para dar conta das principais dificuldades encontradas conforme mostram os estudos: confronto de percepções entre adolescentes e profissionais a respeito do que seja adolescência, fato que faz com que os profissionais tenham dificuldade em estabelecer o vínculo e a aderência tão necessários para que as 
políticas sejam eficazes. É possível que isso ocorra devido a alguns casos de despreparo profissional, sobrecarga dos profissionais que atuam na atenção básica, ausência de projetos de atenção específicos para adolescentes e dificuldade de acesso dos adolescentes aos programas existentes.

Ainda para o âmbito político, estudos apontam estratégias para dar conta de algumas das necessidades mais urgentes. $\mathrm{O}$ trabalho mais indicado e o menos realizado no âmbito das políticas públicas para adolescentes é o da educação, pois o que existe é um grande empenho informativo centrado no uso de anticoncepcionais. Outras propostas continuamente citadas são a atualização e capacitação continuada das equipes para lidar com a temática, a ampliação do acesso dos adolescentes aos serviços educacionais e de saúde e a utilização de um processo educativo na elaboração das políticas que priorize em cada local o desenvolvimento de recursos próprios de cada contexto sempre integrando a família. A utilização dos recursos existentes aparece como uma ideia recorrente na literatura, mas será preciso uma inteligente e corajosa redistribuição de tarefas para a otimização de espaços e de profissionais, sempre articulando as ideias com a realidade dos adolescentes de cada região do País.

O estudo da gravidez na adolescência na sociedade revelou a complexidade desta situação. As publicações científicas abordam implicações educacionais e culturais. Algumas das reflexões propostas remetem-nos aos interesses que estão no limiar da discriminação e que aparecem escondidos atrás da crescente e pesada mobilização em torno da distribuição e do uso de anticoncepcionais, principalmente nas escolas. Apesar destas questões mais ideológicas sob as quais a atenção à gravidez na adolescência está calcada, foi possível constatar que o cuidado às gestantes na esfera social se tem dado através do que podemos chamar de ações coletivas e, de fato, tais ações apresentam-se como as mais indicadas para intervir através da sociedade, pois, em diversos estudos, escola, igreja, mídia e grupo de iguais aparecem como responsáveis por maior socialização e esclarecimento entre os adolescentes. É importante perceber que as ações que deram certo em outros países obtiveram bons resultados a longo prazo e atuaram com base nos chamados fatores de risco e fatores de proteção para gravidez entre adolescentes, especialmente nos fatores de risco quando a adolescente já está grávida. É o caso do programa "Teenager Pregnancy and Parenting Project" (TAPP), em São Francisco, Califórnia que revelou que o envolvimento do pai do bebê nas atividades de educação para a saúde e na preparação para o cuidado infantil levou à maior participação dele no pré-natal, chegando a influenciar o aumento do peso dos bebês ao nascer (Gama e col., 2004). Além destas ações coletivas, não podem ser descartadas as discussões que visem importantes mudanças socioculturais para o padrão de comportamento sexual das sociedades modernas, já que a iniciação sexual precoce precisa ser olhada com maior seriedade e preocupação, porque pode ser considerada um fator de risco pela possibilidade de aumentar o número de casos de gestação em adolescentes.

\section{Considerações Finais}

A partir dos resultados do presente estudo, foi possível compreender o crescente empenho de pesquisadores para refletir sobre o que tem sido feito em relação à gravidez adolescente e propor estratégias mais eficazes para uma melhor atenção e cuidado a essa população. Muitas coisas ainda precisam ser repensadas e não foram encontradas intervenções sólidas e estruturadas em nenhum dos âmbitos estudados. Os fatores de proteção para a evitação ou prevenção da gravidez indesejada na adolescência, tais como maior religiosidade, maior nível de educação e escolaridade dos pais, pais trabalhando fora de casa, além de satisfação pessoal e felicidade, não foram objeto deste trabalho, mas precisam ser tomados como um dos pilares para a construção de estratégias de intervenção nesta temática já que são eles que mostram o que tem sido mais decisivo para os adolescentes que não engravidaram (Michelazzo e col., 2004).

A adolescência parece ocupar um lugar ainda confuso tanto na família, quanto nas políticas públicas e na sociedade. Será preciso vontade e persistência para percorrer um longo caminho rumo aos tão desejados baixos índices de gravidez entre adolescentes. As falhas atuais são evidentes, algumas estratégias para solucioná-las estão propostas. 
A quem cabe fazer? De quem deve ser a iniciativa? Talvez estas sejam questões mais difíceis de responder, pois os estudos indicam sugestões para quase todas as dificuldades. Dessa forma a disposição para implicar-se, para discutir e pôr em prática são as dificuldades mais consistentes encontradas no momento, afinal, intervenções mais incisivas e eficazes são grandes desafios, pois tratam-se de modificações em conceitos, valores, estruturas políticas e comportamentos. Quem está suficientemente forte em nossa cultura para mexer em estruturas tão fundamentais da ordem social?

Serão necessários ainda outros estudos que aprofundem não somente a questão teórica, mas que venham a campo e complementem a visão da comunidade científica a respeito do que tem ocorrido de fato e qual tem sido a eficácia do cuidado à gravidez na adolescência nos três aspectos aqui estudados. Estudos que acompanhem de perto a percepção dos adolescentes grávidos (pais e mães) a respeito dos cuidados e da atenção que recebem da família, programas políticos, de sua cultura e meio social aonde estão inseridos também se fazem necessários para uma visão mais ampla que possibilite intervenções mais assertivas relacionadas à gravidez na adolescência.

\section{Referências}

ALTMANN, H. A. Sexualidade adolescente como foco de investimento político-social. Educação em Revista, Belo Horizonte, v. 46, p. 287-310, 2007.

BARALDI, A. C. P. et al. Gravidez na adolescência: estudo comparativo das usuárias das maternidades públicas e privadas. Revista LatinoAmericana de Enfermagem, Ribeirão Preto, SP, v. 15, p. 799-805, 2005.

BERLOFI, L. M. et al. Prevenção da reincidência de gravidez em adolescentes: efeitos de um programa de planejamento familiar. Acta Paulista de Enfermagem, São Paulo, v. 19, n. 2, p. 196-200, 2006.

BERWANGER, O. et al. Como avaliar criticamente revisões sistemáticas e metanálises? Revista Brasileira de Terapia Intensiva, São Paulo, v. 19, n. 4, p. 475-8o, 2007.
BORGES, A. L. V.; NICHIATA, L. Y. I.; SCHOR, N. Conversando sobre sexo: a rede sociofamiliar como base de promoção da saúde sexual e reprodutiva de adolescentes. Revista LatinoAmericana de Enfermagem, Ribeirão Preto, SP, v. 14, n. 3, p. 422-427, 2006.

CARVACHO, I. E. et al. Fatores associados ao acesso anterior à gestação a serviços de saúde por adolescentes gestantes. Revista de Saúde Pública, São Paulo, v. 42, n. 5, p. 886-94, 2008.

CORRÊA, A. C. P.; FERRIANI, M. G. C. Paternidade na adolescência: um silêncio social e um vazio científico. Revista Gaúcha de Enfermagem, Porto Alegre, v. 27, n. 4, p. 499-505, 2006.

COSTA, M. C. O.; FORMIGLI, V. L. A. Avaliação da qualidade de serviço de saúde para adolescentes. Revista de Saúde Pública, São Paulo, v. 35, n. 2, p. 177-84, 2001.

COSTA, M. C. O. et al. Gravidez na adolescência e co-responsabilidade paterna: trajetória sociodemográfica e atitudes com a gestação e a criança. Ciência \& Saúde Coletiva, Rio de Janeiro, v. 10, n. 3, p. 719-27, 2005.

DAMIAN, F. E. Gravidez na adolescência: a quem cabe prevenir? Revista Gaúcha de Enfermagem, Porto Alegre, v. 24, n. 2, 2003, editorial.

DIAS, A. C. G.; GOMES, W. B. Conversas sobre sexualidade na família e gravidez na adolescência: a percepção dos pais. Estudos de Psicologia, Natal, v. 4, n. 1, p. 79-106, 1999.

BRASIL. Lei no 8.069. Estatuto da Criança e do Adolescente. Brasília, DF, 199o. Disponível em <http://www.planalto.gov.br/ccivil_o3/leis/ 18069compilado.htm>. Acesso em: 12 ago. 2010.

FERRARI, R. A. P.; THOMSON, Z.; MELCHIOR, R. Adolescência: ações e percepção dos médicos e enfermeiros do Programa Saúde da Família. Interface - Comunicação, Saúde, Educação, Botucatu, SP, v. 12, n. 25, p. 387-400, 2008.

GAMA, S. G. N. et al. Fatores associados à assistência pré-natal precária em uma amostra de puérperas adolescentes em maternidades do Município do Rio de Janeiro. Cadernos de Saúde Pública, Rio de Janeiro, v. 20, n. 1, p. 101-11, 2004. 
GODINHO, R. A. et al. Adolescentes e grávidas: onde buscam apoio? Revista Latino-Americana de Enfermagem, Ribeirão Preto, SP, v. 8, n. 2, p. 25-32, 2000.

GONÇALVES, S. D.; PARADA, C. M. G. L.;

BERTONCELLO, N. M. F. Percepção de mães adolescentes acerca da participação paterna na gravidez, nascimento e criação do filho. Revista Escola de Enfermagem USP, São Paulo, v. 35, n. 4, p. 406-13, 2001.

HEILBORN, M. L. et al. Aproximações sócioantropológicas sobre a gravidez na adolescência. Horizonte Antropológico, Porto Alegre, v. 8, n. 17, p. 13-45, 2002.

LIMA, C. T. B. et al. Percepções e práticas de adolescentes grávidas e de familiares em relação à gestação. Revista Brasileira de Saúde MaternoInfantil, Recife, v. 4, n. 1, p. 71-83, 2004.

LIRA, J. B.; DIMENSTEIN, M. Adolescentes avaliando um projeto social em uma unidade básica de saúde. Psicologia em Estudo, Maringá, v. 9, n. 1, p. 37-45, 2004.

MICHELAZZO, D. et al. Indicadores sociais de grávidas adolescentes: estudo caso-controle. Revista Brasileira de Ginecologia e Obstetrícia, São Paulo, v. 26, n. 8, p. 633-9, 2004.

MONTEIRO, C. F.. A violência intra-familiar contra adolescentes grávidas. Revista Brasileira de Enfermagem, Brasília, v. 6o, n. 4, p. 373-6, 2007.

MOREIRA, T. M. et al. Conflitos vivenciados pelas adolescentes com a descoberta da gravidez. Revista Escola de Enfermagem da USP, São Paulo, v. 42, n. 2, p. 312-20, 2008.

OLIVEIRA, M. W. Gravidez na adolescência: dimensões do problema. Cadernos CEDES, Campinas, SP, v. 19, n. 45, p. 48-70, 1998.

ORLANDI, R.; TONELI, M. J. F. Adolescência e paternidade: sobre os direitos de criar projetos e procriar. Psicologia em Estudo, Maringá, PR, v. 13, n. 2, p. 317-26, 2008.

OTANI, M. A. P.; BARROS, N. F. A medicina integrativa e a construção de um novo modelo na saúde. Ciência \& Saúde Coletiva, Rio de Janeiro, v. 16, n. 3, p. 1801-11, 2011.
PANTOJA, A. L. N. "Ser alguém na vida": uma análise sócio-antropológica da gravidez/ maternidade na adolescência, em Belém do Pará, Brasil. Cadernos de Saúde Pública, Rio de Janeiro, v. 19, n. 2, p. 335-43, 2003.

PAPALIA, D. E.; OLDS, S. W. ; FELDMAN, R. D. Desenvolvimento humano. Porto Alegre: Artmed, 2006.

SANTOS, S. R.; SCHOR, N. Vivências da maternidade na adolescência precoce. Revista de Saúde Pública, São Paulo, v. 37, n. 1, p. 15-23, 2003.

SILVA, L.; TONETE, V. L. P. A gravidez na adolescência sob a perspectiva dos familiares: compartilhando projetos de vida e cuidado. Revista Latino-Americana de Enfermagem, Ribeirão Preto, SP, v. 14, n. 2, p. 199-206, 2006.

SIMÕES, V. M. F. et al. Características da gravidez na adolescência em São Luís, Maranhão. Revista de Saúde Pública, São Paulo, v. 37, n. 5, p. 559-65, 2003.

SIQUEIRA, M. J. T. et al. Profissionais e usuárias(os) adolescentes de quatro programas públicos de atendimento pré-natal da região da grande Florianópolis: onde está o pai? Estudos de Psicologia, Natal, v. 7, n. 1, p. 65-72, 2002.

SOUZA, I. F. Gravidez de adolescência: uma questão social. Adolescência Latino-Americana, Belo Horizonte, MG, v. 3, n. 2, p. o-o, 2006.

VIEIRA, L. M. et al. Reflexões sobre a anticoncepção na adolescência no Brasil. Revista Brasileira e Saúde Materno Infantil, Recife, v. 6, n. 1, p. 135-40, 2006.

YAZLLE, M. E. H. D. Gravidez na adolescência. Revista Brasileira de Ginecologia e Obstetrícia, São Paulo, v. 28, n. 8, p. 443-5, 2006.

Recebido em: 15/12/2010 Reapresentado em: 23/02/2012 Aprovado em: 08/05/2012 